TRANSACTIONS OF THE

AMERICAN MATHEMATICAL SOCIETY

Volume 353, Number 3, Pages 963-972

S 0002-9947(00)02669-6

Article electronically published on September 15, 2000

\title{
A BOUNDING QUESTION FOR ALMOST FLAT MANIFOLDS
}

\author{
SHASHIDHAR UPADHYAY
}

\begin{abstract}
We study bounding question for almost flat manifolds by looking at the equivalent description of them as infranilmanifolds $\Gamma \backslash L \rtimes G / G$. We show that infranilmanifolds $\Gamma \backslash L \rtimes G / G$ bound if $L$ is a 2-step nilpotent group and $G$ is finite cyclic and acts trivially on the center of the nilpotent Lie group $L$.
\end{abstract}

\section{INTRODUCTION}

Let $M^{n}$ be a closed (connected) smooth manifold of dimension $n$. We say that $M^{n}$ bounds if there exists a compact $(n+1)$-dimensional manifold with boundary $W^{n+1}$ such that the boundary of $W^{n+1}$ is diffeomorphic to $M^{n}$. By a well known theorem of Thom, a smooth manifold bounds if and only if its Stiefel-Whitney numbers vanish.

Hamrick and Royster [5] proved that "Any closed connected Riemannian flat manifold bounds".

Let us recall Gromov's almost flat manifolds [4. A closed connected smooth manifold is called an "almost flat manifold" if there exists a sequence of Riemannian metrics $g_{i}()$, where $i=1,2,3, \ldots$, such that

- $\lim _{i \rightarrow \infty} K\left(M^{n}, g_{i}\right)=0$, and

- $\left\{d\left(M^{n}, g_{i}\right): i=1,2,, \ldots\right\}$ has a finite upper bound.

Here $K\left(M^{n}, g_{i}\right)$ is the maximum of the sectional curvatures of $\left(M^{n}, g_{i}\right)$. Gromov proved [4] the following important theorem about almost flat manifolds.

Theorem 1.1 (Gromov). Let $M^{n}$ be an almost flat manifold. Then $M$ has a finite sheeted regular cover $M^{\prime}$ which is diffeomorphic to a nilmanifold.

Corollary 1.2. $M$ is aspherical, and $\pi_{1}(M)=\Gamma$ fits into the exact sequence

$$
1 \longrightarrow N \longrightarrow \Gamma \longrightarrow G \longrightarrow 1,
$$

where $N$ is finitely generated torsion-free nilpotent group, $G$ a finite group.

Recall that a nilmanifold is a quotient of a simply connected nilpotent Lie group by a discrete co-compact subgroup. Note that since nilmanifolds are parallelizable, this result of Gromov implies that the rational Pontryagin classes of $M$ vanish. This was the motivation of Farrell and Zdravkovska's conjecture [2]:

Conjecture 1.3. If $M^{n}$ is an almost flat manifold, then there exists a compact smooth manifold $W^{n+1}$ such that $\partial W^{n+1}=M^{n}$, i.e., the boundary of $W^{n+1}$ is $M^{n}$.

Received by the editors August 3, 1999.

1991 Mathematics Subject Classification. Primary 57R19, 57R20; Secondary 55N22.

Key words and phrases. Almost flat manifolds, infranilmanifolds, Stiefel-Whitney numbers. 
A connected smooth manifold $X$ is called an infranilmanifold if it is diffeomorphic to a double coset space $\Gamma \backslash L \rtimes G / G$, where $L$ is a (connected) simply connected nilpotent Lie group, $L \rtimes G$ is the semi-direct product with respect to a faithful representation of a finite group $G$ into $\operatorname{Aut}(L)$, and $\Gamma$ is a discrete co-compact, torsion-free subgroup of $L \rtimes G$. In such a case $G$ is called the holonomy group of the infranilmanifold $X$.

By Corollary 1.2, $\pi_{1}\left(M^{n}\right)=\Gamma$ is a finitely generated torsion-free virtually nilpotent group, then by, Mal'cev's work (cf. [7] or 8], Theorem 2.18), $M^{n}$ can be chosen to be an infranilmanifold (up to homotopy equivalence). This is done as follows.

Let $N$ be a discrete, nilpotent, finite index subgroup of $\Gamma$. Without loss of generality we can assume that $N$ is a normal subgroup of $\Gamma$. By Mal'cev's theorem (8), Theorem 2.18) there exists a (connected) simply connected nilpotent Lie group $L$ which contains $N$ as a lattice. We have the following pushout diagram:

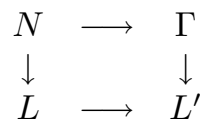

Here $L^{\prime}$ is the pushout of the diagram. It is a fact that $L^{\prime}$ splits as $L \rtimes G$ (see [2]). It is clear now that $\Gamma \backslash L \rtimes G / G$ has fundamental group $\Gamma$.

Since any infranilmanifold supports an almost flat structure, the homotopy type of an aspherical manifold is determined by its fundamental group, and StiefelWhitney classes are homotopy invariants, we have that Conjecture 1.3 is equivalent to the following.

Conjecture 1.4. Let $M^{n}=\Gamma \backslash L \rtimes G / G$, where $\Gamma, L$ and $G$ are as above. Then $M^{n}$ bounds.

Remark 1.5. Note that it is sufficient to prove the above conjecture for $G$ whose order is a power of 2 . This is because Stiefel-Whitney numbers are invariant under odd-sheeted covering.

Let $Z(L)$ denote the center of $L$. Farrell and Zdravkovska [2] proved Conjecture 1.4 in the case when $G$ acts effectively on the center $Z(L)$ (by direct extensions of the proof of Hamrick and Royster in the flat manifolds case), and also for the case $G=\mathbb{Z}_{2}$ when $G$ does not act effectively on $Z(L)$ (which is the same as acting trivially on $Z(L)$ ). In this paper we extend the result of [2]. Recall that a 2-step nilpotent group $L$ is a nilpotent group such that $[L, L] \subset Z(L)$. ([L,L] denotes the commutater subgroup.) Our main theorem is:

Theorem 1.6. Conjecture 1.4 is true if $G$ is finite cyclic and $L$ is any (connected) simply connected 2-step nilpotent Lie group such that $G$ acts trivially on the center of $L$.

\section{Preliminaries}

In this section we recall a standard way to construct $\left(\mathbb{Z}_{2}\right)^{k}$ actions on infranilmanifolds. We will also recall theorems of Stong [9] and of Kosniowski and Stong [6]. Their theorem gives a way to study bounding questions for any manifold with $\left(\mathbb{Z}_{2}\right)^{k}$ actions in terms of fixed points and its normal bundle.

The following construction is originally due to M. W. Gordon [3], who con-

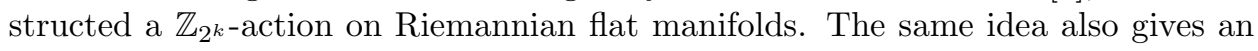


action on infranilmanifolds, and was used by Farrell and Zdravkovska [2]. In view of Remark 1.5, from now on $G$ will be a finite group whose order is a power of 2 .

Let $\Gamma$ be a finitely generated torsion free group containing a nilpotent subgroup $N$ of finite index a power of 2 so that the sequence

$$
1 \longrightarrow N \longrightarrow \Gamma \longrightarrow G \longrightarrow 1
$$

is exact. There is a regular covering $p: \tilde{M} \longrightarrow M$, where $\tilde{M}=N \backslash L$, defined by $p(N x)=\Gamma x G$ for $x \in L$. The group $G$ acts freely on $\tilde{M}$ as the group of covering transformations by

$$
g(N x)=N \gamma x g^{-1},
$$

where $\gamma$ is any element of $\Gamma$ such that $\gamma x g^{-1} \in L$. It is easy to see that this action is independent of the choice of $\gamma$. Let $Z(L)$ denote the center of $L$. Since $L$ is nilpotent, it follows that $Z(L) \neq(1)$ and $Z(N)$ is a co-compact subgroup of $Z(L)$. (To see that $Z(N) \subset Z(L)$, use Mal'cev's Rigidity Theorem, [8], Theorem 2.11.) $Z(L)$ acts on $L$ by multiplication; hence $Z(N) \backslash Z(L)$ acts on $N \backslash L=\tilde{M}$. Let $\Sigma$ denote the subgroup of $Z(N) \backslash Z(L)$ consisting of elements of order 2 ; and $\Sigma_{G}$ the subgroup of elements in $\Sigma$ fixed under the conjugation action of $G$ on $\Sigma$. Since $G$ is a 2-group by assumption, $\Sigma_{G}$ is nontrivial: the orbits of $G$ in $\Sigma$ consist of either one element or an even number of elements, $1 \in \Sigma$ is the single element in the orbit, $|\Sigma|$ is even, hence there is at least one more element of $\Sigma$ fixed under $G$. It can be easily checked that the covering action of $G$ on $\tilde{M}$ and the multiplication action of $\Sigma_{G}$ on $\tilde{M}$ commute, and hence the action of $\Sigma_{G}$ on $\tilde{M}$ passes to an action of $\Sigma_{G}$ on $M$ :

$$
Z(N) z(\Gamma x G)=\Gamma z x G
$$

for $x \in L, z \in Z(L)$ such that $2 z \in Z(N)$ and $Z(N) g z g^{-1}=Z(N) z$ for all $g \in G$. If there is no fixed point of $\Sigma_{G}$ on $M$, then $M$ bounds by a result of Conner and Floyd (cf. 11, Theorem 30.1). Let $\Gamma x G$ be a fixed point. Then there is an injective homomorphism (depending on the choice of the fixed point) $\phi: \Sigma_{G} \longrightarrow G$ defined by putting $\phi(z)=g$ if and only if $z(N x)=g(N x)$. This $g$ is determined uniquely. From now on we will always assume that $\Sigma_{G}$ has a fixed point on $M$.

Now we recall theorems of Stong [9] and Kosniowski-Stong [6].

Let $M$ be a closed manifold and let $\left\{\xi_{i} \longrightarrow M\right\}, i \geq 1$, be vector bundles over $M$. We shall say that $\left\{\xi_{i} \longrightarrow M\right\}, i \geq 1$, bounds as a bundle space if there is a compact manifold $W$ with boundary $\partial W$ and vector bundles $\left\{\eta_{i} \longrightarrow W\right\}, i \geq 1$, satisfying

(i) $M=\partial W$, and

(ii) $\left.\eta_{i}\right|_{\partial W} \longrightarrow \partial W$ as $\xi_{i} \longrightarrow M$ for $i \geq 1$.

An action of $\left(\mathbb{Z}_{2}\right)^{k}$ on the bundle space $\left\{\xi_{i} \longrightarrow M\right\}, i \geq 1$, shall mean a smooth action on $M$ covered by actions on the total spaces of each of the $\xi_{i}$ which are linear on the fibers.

The following theorem is due to Stong [9], but in the following form it is stated and proved in [5].

Theorem 2.1 (Stong). Let $M$ be a closed manifold with vector bundles $\left\{\xi_{i} \longrightarrow\right.$ $M\}, i \geq 1$. Suppose $\left(\mathbb{Z}_{2}\right)^{k}$ acts on the bundle space $\left\{\xi_{i} \longrightarrow M\right\}, i \geq 1$. Let $F$ be the fixed set of the action in $M$, let $\left\{\xi_{i j} \longrightarrow F\right\}, 2^{k} \geq j \geq 1$, be the eigenbundles of $\left.\xi_{i}\right|_{F} \longrightarrow F$ for $i \geq 1$, and let $\left\{\nu_{j} \longrightarrow F\right\}, 2^{k}-1 \geq j \geq 1$, be the normal eigenbundles 
in $M$. Then the bundle space $\left\{\xi_{i} \longrightarrow M\right\}, i \geq 1$, bounds if $\left\{\xi_{i j}, \nu_{j} \longrightarrow F\right\}$ bounds as a bundle space.

By Conner and Floyd [1, to show that $\left\{\xi_{i} \longrightarrow M\right\}, i \geq 1$, bounds it is sufficient to show that $\langle w(M, \xi),[M]\rangle=0$, where $w(M, \xi)$ is monomial in the Stiefel-Whitney classes of $M$ and $\xi_{i}$ 's of total dimension equal to the dimension of $M$, and $[M]$ is the fundamental homology class of $M$. If $M$ is not connected, then it is required that the rank of $\xi_{i}$ over each component of $M$ be the same. In our context the eigenbundles over different components of the fixed point set will not have the same rank in general. But we can always decompose our fixed point set as the disjoint union of two closed subset so that eigenbundles restricted to each of these closed subset have a fixed rank, and hence this result of Conner and Floyd can be applied to these closed subsets. To effectively use this result of Conner and Floyd in our context we need a reformulated version of the above theorem in the case when $k=1$.

Theorem 2.2. Let $M$ be a closed manifold with vector bundles $\left\{\xi_{i} \longrightarrow M\right\}, i \geq 1$. Suppose $\left(\mathbb{Z}_{2}\right)$ acts on the bundle space $\left\{\xi_{i} \longrightarrow M\right\}, i \geq 1$. Let $F=F_{1} \cup F_{2}$ be the fixed point set of the $\mathbb{Z}_{2}$-action on $M$, where $F_{1}$ and $F_{2}$ are closed disjoint subsets of $F$. Let $\nu_{1}$ and $\nu_{2}$ be the normal bundles of $F_{1}$ and $F_{2}$ respectively. Let $\xi_{i}{ }^{+}$ and $\xi_{i}{ }^{-}$be the eigenbundles over $F$. Define $\xi_{i j}{ }^{+}=\xi_{i F_{j}}^{+}$and $\xi_{i j}{ }^{-}=\xi_{i \mid F_{j}}^{-}, j=$ 1,2. Then the bundle space $\left\{\xi_{i} \longrightarrow M\right\}, i \geq 1$, bounds if $\left\{F_{1}, \nu_{1}, \xi_{i 1}{ }^{+}, \xi_{i 1}{ }^{-}\right\}$and $\left\{F_{2}, \nu_{2}, \xi_{i 2}{ }^{+}, \xi_{i 2}{ }^{-}\right\}$bound.

Proof. We adapt the proof given by Hamrick and Royster.

Case $1 . \mathbb{Z}_{2}=\langle t\rangle$ acts freely.

Then $\xi_{i}$ is the restriction to the boundary $M$ of the base space of the bundle

$$
\xi_{i} \times[-1,1] /(v, r) \sim(t v,-r) \longrightarrow M \times[-1,1] /(m, r) \sim(t m,-r) .
$$

Case 2. The $\mathbb{Z}_{2}$ action is not free.

By Case 1 it is sufficient to show that the bundle space $\left\{\xi_{i} \longrightarrow M\right\}, i \geq 1$, is equivariantly cobordant to another bundle space where $\mathbb{Z}_{2}$ acts freely.

By hypothesis there exist $W_{1}, W_{2}, \mu_{1}, \mu_{2}$ and $\eta_{i 1}^{+}, \eta_{i 1}^{-}, \eta_{i 2}^{+}, \eta_{i 2}^{-}$such that $\partial W_{j}=$ $F_{j}, j=1,2, \mu_{j} \longrightarrow W_{j}$ and $\eta_{i j}^{ \pm} \longrightarrow W_{j}, j=1,2$, such that $\eta_{i j}^{ \pm} \mid F_{j}=\xi_{i j}{ }^{ \pm}, j=1,2$, and $\mu_{j} \mid F_{j}=\nu_{j}, j=1,2$. Let $D\left(\mu_{1}\right)$ and $D\left(\mu_{2}\right)$ denote the disc bundles of $\mu_{1}$ and $\mu_{2}$ respectively. Note that $D\left(\mu_{j}\right)_{\mid F_{j}}=\nu_{j}, j=1,2$. Consider the cobordism

$$
M \times[0,1] \cup\left(D\left(\mu_{1}\right) \cup D\left(\mu_{2}\right)\right) / \sim
$$

obtained by gluing $M \times 1$ and $\left(D\left(\mu_{1}\right) \cup D\left(\mu_{2}\right)\right)$ along $D\left(\nu_{1}\right) \cup D\left(\nu_{2}\right)$. (Here we regard $D\left(\nu_{1}\right)$ and $D\left(\nu_{2}\right)$ as tubular neighborhoods of $F_{1}$ and $F_{2}$.) There is an involution on this cobordism induced by the involution on $M$ and scalar multiplication by -1 in the fibers of $D\left(\mu_{1}\right)$ and $D\left(\mu_{2}\right)$. Note that there are no fixed points in the boundary of the cobordism except at $M \times 0$.

Let $\pi_{j}: D\left(\mu_{j}\right) \longrightarrow W_{j}, j=1,2$, be the projection. We can adjoin the bundle $\xi_{i} \times[0,1] \longrightarrow M \times[0,1]$ to $\left(\pi_{1}{ }^{*}\left(\eta_{i 1}^{+} \oplus \eta_{i 1}^{-}\right) \cup \pi_{2}{ }^{*}\left(\eta_{i 2}^{+} \oplus \eta_{i 2}^{-}\right)\right) \longrightarrow\left(D\left(\mu_{1}\right) \cup D\left(\mu_{2}\right)\right)$ to obtain a bundle over the cobordism by means of equivalence:

$$
\xi_{i} \times\{1\}_{\mid D\left(\nu_{j}\right)} \simeq\left(\pi_{j \mid D\left(\nu_{j}\right)}\right)^{*}\left(\xi_{i j}^{+} \oplus \xi_{i j}^{-}\right)=\left(\pi_{j}^{*}\left(\eta_{i j}^{+} \oplus \eta_{i j}^{-}\right)\right), \quad j=1,2 .
$$

There is a bundle involution inherited from the involution on $\xi_{i} \times[0,1]$ and scalar multiplication by -1 in the fibers of $\eta_{i j}{ }^{-}, j=1,2$. This makes the bundle space 
$\left\{\xi_{i} \longrightarrow M\right\}, i \geq 1$, cobordant to a bundle space with free $\mathbb{Z}_{2}$ action. This completes the proof.

\section{Proof of Theorem 1.6}

Now we specialize to the case where $G$ is isomorphic to $\mathbb{Z}_{2^{s}}, s>1$, cyclic group of order $2^{s}$ generated by, say, $g$. (The case $s=1$ has already been done by Farrell and Zdravkovska, as mentioned earlier.) Also, we are assuming that $G$ acts trivially on the center $Z(L)$ of $L$. Since $G$ acts trivially on $Z(L)$, we note that $\Sigma_{G}=\Sigma$. As mentioned earlier in the Preliminaries, $\Sigma_{G}$ acts on $M=\Gamma \backslash L \rtimes G / G$ and is assumed to have a fixed point; but then $\Sigma_{G}$ has to map injectively into $G=\mathbb{Z}_{2^{s}}$. This forces $\Sigma_{G}$ to be a cyclic group of order 2 generated by, say, $\bar{b}_{1}$ and under the map $\phi: \Sigma_{G} \longrightarrow G, \phi\left(\bar{b}_{1}\right)=g^{2^{s-1}}$. Note that this also forces $Z(L)$ to be 1-dimensional. We have the covering map

$$
G \longrightarrow \tilde{M} \longrightarrow M
$$

Note that $Z(N) \backslash Z(L)$ acts on $N \backslash L$ by coset multiplication, i.e, $(Z(N) b)(N x)=$ $N b x$. From now on we will denote $Z(N) b$ as $\bar{b}$. We note at this point that since $G$ acts trivially on $Z(L)$, not only $\Sigma_{G}$ but all of $Z(N) \backslash Z(L)$ acts on $M$. To see this we need to show that $g \bar{b}(N x)=\bar{b} g(N x)$ for $g \in G$ and $\bar{b} \in Z(N) \backslash Z(L)$. By definition $g \bar{b}(N x)=g(N b x)=N \gamma b x g^{-1}$, where $\gamma \in \Gamma$ is such that $\gamma \mapsto g$ in the exact sequence $N \longrightarrow \Gamma \longrightarrow G$. Since $\gamma$ also belongs to $L^{\prime}$, it can be written as $l . g$ in $L^{\prime}$ for some $l \in L$. This says that $N \gamma b x g^{-1}=N l g b x g^{-1}=N b l g x g^{-1}=$ $\bar{b} N \gamma x g^{-1}=\bar{b} g(N x)$, because $b$ commutes with $g$ as well as with $l$.

Choose $b_{j} \in Z(L), j=1,2, \ldots, s$, such that $\bar{b}_{1}^{2}=i d$ and $\bar{b}_{j}^{2}=\bar{b}_{j-1}$. Note that $\bar{b}_{s}$ generates a cyclic group of order $2^{s}$ in $Z(N) \backslash Z(L)$. Define $\alpha_{j}=g^{2^{s-j}}$. For $k=1,2, \ldots 2^{j-1}$, define $E_{j}=\bigcup_{k} E_{j}^{k}$, where $E_{j}^{k}$ is defined as follows:

$$
E_{j}^{k}=\left\{N x \in N \backslash L: \bar{b}_{j}(N x)=\alpha_{j}{ }^{2 k-1}(N x)\right\} .
$$

This means that, for example,

$$
E_{1}=\left\{N x \in N \backslash L: \bar{b}_{1}(N x)=\alpha_{1}(N x)\right\}
$$

and

$$
E_{2}=\left\{N x \in N \backslash L: \bar{b}_{2}(N x)=\alpha_{2}(N x) \text { or } \bar{b}_{2}(N x)=\alpha_{2}{ }^{3}(N x)\right\} .
$$

Note that $E_{1}$ is non-empty, since we are assuming that $\Sigma_{G}=\left\langle\bar{b}_{1}\right\rangle$ has a fixed point in $M$. This is seen as follows. Let $\Gamma x G \in M$ be a fixed point of $\bar{b}_{1}, x \in L$. This means that $N b_{1} x=g^{t}(N x)$ for some $t$. Applying $\bar{b}_{1}$ again on both sides, we get $N x=g^{2 t}(N x)$. But $G$-action is a covering action, so it cannot have any fixed point. This means $g^{2 t}=i d$, and hence $g^{t}=g^{2^{s-1}}=\alpha_{1}$.

Also note that $E_{j+1} \subset E_{j}, j=1,2, \ldots, s-1$. Let us also assume that $E_{s}$ is non-empty. If $E_{s}$ or any of the preceding $E_{j}, j \leq s$, is empty then the proof in this case is simpler, which will be evident from the proof of the case when $E_{s}$ is non-empty. Since we are assuming that $E_{s}$ is non-empty, without loss of generality we can assume that $E_{s}^{1}$ is non-empty.

Let $N x \in E_{s}^{1}$. This means $N b_{s} x=\alpha_{s}(N x)=N \gamma x \alpha_{s}^{-1}$, and so $\gamma x \alpha_{s}^{-1}=n b_{s} x$ for some $n \in N$, which in turn implies $n^{-1} \gamma=b_{s} x \alpha_{s} x^{-1} \in \Gamma$. By changing $G$ to $x G x^{-1}$ in the splitting of $L^{\prime}$, we can assume that $b_{s} \alpha_{s} \in \Gamma$. This last condition $\left(b_{s} \alpha_{s} \in \Gamma\right)$ implies that $\alpha_{s}=g$ acts on $N$. This is seen as follows. Let $n \in N$; then $b_{s} \alpha_{s} n \alpha_{s}^{-1} b_{s}^{-1} \in \Gamma$. But since $b_{s} \in Z(L)$, we have $b_{s} \alpha_{s} n \alpha_{s}^{-1} b_{s}^{-1}=\alpha_{s} n \alpha_{s}^{-1} \in \gamma$. 
However, $\alpha_{s} n \alpha_{s}^{-1}$ maps to $e \in G$, so it must lie in $N$. Since $\alpha_{s}$ acts on $N$, all $\alpha_{j}, j \leq s$, act on $N$.

Lemma 3.1. Each $E_{j}$ is a finite union of closed connected nilmanifolds, and all components of $E_{j}$ have the same dimension.

Proof. Let us first consider the case $j=1$.

Recall that $E_{1}=\left\{N x \in N \backslash L: N b_{1} x=\alpha_{1}(N x)\right\}$. By definition of the covering action on $N \backslash L$ we have $\alpha_{1}(N x)=N \gamma x \alpha_{1}$, where $\gamma \in \Gamma$ is such that $\gamma \mapsto \alpha_{1}$ in the exact sequence

$$
1 \longrightarrow N \longrightarrow \Gamma \longrightarrow G \longrightarrow 1
$$

Since $b_{s} \alpha_{s} \in \Gamma$, and $b_{s}, \alpha_{s}$ commute (because the $G$-action on the center is trivial), we also have that $\left(b_{s} \alpha_{s}\right)^{s}=b_{1} \alpha_{1} \in \Gamma$. Hence $\gamma$ can be chosen to be $b_{1} \alpha_{1}$. This implies that if $N x \in E_{1}$ then $N b_{1} x=N b_{1} \alpha_{1} x \alpha_{1}$, and hence $N x=N \alpha_{1} x \alpha_{1}$. In other words, $E_{1}$ is the fixed point set of the conjugation action of $\alpha_{1}$ on $N \backslash L$ induced by the conjugation action of $\alpha_{1}$ on $L$. (Note that $\alpha_{1}(N) \subset N$.) Let $p: L \longrightarrow N \backslash L$ be the quotient map. Then $p^{-1}\left(E_{1}\right)=\bigcup_{n \in N} E_{1}^{(n)}$, where $E_{1}^{(n)}$ is defined as follows:

$$
E_{1}^{(n)}=\left\{x \in L: \alpha_{1}(x)=n x, n \in N\right\} .
$$

Note that $E_{1}^{(n)}$ does not have to be non-empty for all $n \in N$. (In fact a necessary condition for $E_{1}^{(n)}$ to be non-empty is $\alpha_{1}(n)=n^{-1}$.) In particular, $E_{1}^{(e)}=L^{\alpha_{1}}$, the Lie subgroup of $L$ fixed under the homomorphism $\alpha_{1}$. Now we can write $E_{1}=\bigcup_{n \in N} p\left(E_{1}^{(n)}\right)$. It is easy to see that the $p\left(E_{1}^{(n)}\right)$ 's are components of $E_{1}$. Two main points to note are that the $E_{1}^{(n)}$, s are components of $p^{-1}\left(E_{1}\right)$, and also that if $p\left(E_{1}^{(n)}\right) \cap p\left(E_{1}^{(m)}\right) \neq \emptyset$, then $p\left(E_{1}^{(n)}\right)=p\left(E_{1}^{(m)}\right)$. This union is finite because $E_{1}$ is a closed subset of a compact manifold $N \backslash L$, and hence a compact set.

Now we show that each non-empty $p\left(E_{1}^{(n)}\right)$ can be identified with a nilmanifold. To show this it is sufficient to give a transitive action of a nilpotent group. This action is given as follows. Let $x \in L^{\alpha_{1}}$ and $N y \in p\left(E_{1}^{(n)}\right)$, and define $x(N y)=$ $N y x^{-1}$. Let $N y_{1}, N y_{2} \in p\left(E_{1}^{(n)}\right), y_{1}, y_{2} \in E_{1}^{(n)}$. Then $N y_{1}=N y_{2}\left(y_{1}^{-1} y_{2}\right)^{-1}$ and $\left(y_{1}^{-1} y_{2}\right) \in L^{\alpha_{1}}$. This shows that the action is transitive. The isotropy group of this action at $N x_{o}, x_{o} \in E_{1}^{(n)}$ is given by $x_{o}^{-1} S x_{o}$, where $S=\left\{m \in N: m E_{1}^{n}=E_{1}^{n}\right\}$. This completes the proof of Lemma 3.1 for $E_{1}$.

For $j>1$, recall that $E_{j}=\bigcup_{k} E_{j}^{k}, k=1,2, \ldots, 2^{j-1}$, where $E_{j}^{k}=\{N x \in N \backslash L$ : $\left.\bar{b}_{j}(N x)=\alpha_{j}^{2 k-1}(N x)\right\}$. To prove Lemma 3.1, consider the finite sheeted covering $N \backslash L \longrightarrow N^{\prime} \backslash L$, where $N^{\prime}=\left\langle N, b_{j-1}\right\rangle$, the subgroup generated by $N$ and $b_{j-1}$. Let $E_{j}^{\prime}$ be the fixed point of the conjugation action of $\alpha_{j}$ on $N^{\prime} \backslash L$ induced by the conjugation action of $\alpha_{j}$ on $L$. Then $E_{j}^{\prime}$ is a finite disjoint union of nilmanifolds, all having the same dimension. The argument for this is the same as it was for $E_{1}$. Next, note that $E_{j}$ is a finite sheeted covering of $E_{j}^{\prime}$. Since a finite sheeted covering of a nilmanifold is a nilmanifold, $E_{j}$ is a finite disjoint union of nilmanifolds. This completes the proof of the Lemma 3.1.

Since the covering action of $G$ on $\tilde{M}=N \backslash L$ and coset multiplication by an element of $Z(N) \backslash Z(L)$ commute, the action of each $\bar{b}_{j}$ on $\tilde{M}$ descends to act to on $M$. Let $M^{b_{j}}$ denote the fixed point set of the $\bar{b}_{j}$-action on $M, j=1,2, \ldots, s$. Note that $M^{b_{j+1}} \subset M^{b_{j}}$. We have the following lemma concerning the $M^{b_{j}}$ 's. 
Lemma 3.2. Each $M^{b_{j}}$ is a finite (disjoint) union of infranilmanifolds, and each component has holonomy of order at most $2^{s-j}$.

Proof. Let $q: \tilde{M} \longrightarrow M$ denote the covering map. Then it is easy to see that $q^{-1}\left(M^{b_{j}}\right)=E_{j}$. In other words, $q_{\mid E_{j}}: E_{j} \longrightarrow M^{b_{j}}$ is a covering map with $G$ as covering transformation.

Once again let us consider the case $j=1$ first.

Let $M_{0}^{b_{1}}$ be a component of $M^{b_{1}}$. Since $q_{\mid E_{1}}: E_{1} \longrightarrow M^{b_{1}}$ is a covering map, there is a component of $E_{1}$, say $p\left(E_{1}^{(n)}\right)$, which maps onto $M_{0}^{b_{1}}$. It is easy to check that $p\left(E_{1}^{(n)}\right)$ is invariant under the covering action of $\alpha_{1}$. Hence $\left(\alpha_{1}\right) \backslash p\left(E_{1}^{(n)}\right) \longrightarrow$ $M_{0}^{b_{1}}$ is a covering map with covering transformation group a cyclic group of order at most $2^{s-1}$. Now we will show that $\left(\alpha_{1}\right) \backslash p\left(E_{1}^{(n)}\right)$ is also a nilmanifold, which will complete the proof for the $M^{b_{1}}$ case.

Recall that in Lemma 3.1 we constructed a transitive action of $L^{\alpha_{1}}$ on $p\left(E_{1}^{(n)}\right)$. As mentioned earlier, the isotropy group at $N x_{o} \in p\left(E_{1}^{(n)}\right), x_{o} \in E_{1}^{n}$, is $S_{o}=$ $x_{o}^{-1} S x_{o}$, where $S=\left\{m \in N: m E_{1}^{n}=E_{1}^{n}\right\}$. Let $\phi_{1}: S_{o} \backslash L^{\alpha_{1}} \longrightarrow p\left(E_{1}^{(n)}\right)$ be the diffeomorphism $\phi_{1}(N x)=N x_{o} x^{-1}$. Note that $\bar{b}_{1}$ acts on both the spaces in a natural way, and the diffeomorphism $\phi_{1}$ commutes with this action of $\bar{b}_{1}$, hence induces a diffeomorphism of $\left\langle S_{o}, b_{1}\right\rangle \backslash L^{\alpha_{1}}$ and $\left(\bar{b}_{1}\right) \backslash p\left(E_{1}^{(n)}\right)$, where $\left\langle S_{o}, b_{1}\right\rangle$ denotes the subgroup of $L^{\alpha_{1}}$ generated by $S_{o}$ and $b_{1}$. Since $b_{1}^{2} \in S_{o},\left\langle S_{o}, b_{1}\right\rangle$ is still a lattice in $L^{\alpha_{1}}$ and hence $\left(\bar{b}_{1}\right) \backslash p\left(E_{1}^{(n)}\right)$ is a nilmanifold. But since $p\left(E_{1}^{(n)}\right) \subset E_{1}$, we have $\left(\bar{b}_{1}\right) \backslash p\left(E_{1}^{(n)}\right)=\left(\alpha_{1}\right) \backslash p\left(E_{1}^{(n)}\right)$. This completes the proof for $M^{b_{1}}$.

For $j>1$, note that $E_{j} \longrightarrow M^{b_{j}}$ is a $2^{s}$-sheeted covering and $E_{j}^{\prime} \longrightarrow M^{b_{j}}$ is $2^{s-j+1}$ sheeted covering. Now we use the same argument as in the $E_{1}$ case to claim that $E_{j}^{\prime \prime}=\left\langle\alpha_{j}\right\rangle \backslash E_{j}^{\prime}=\left\langle b_{j}\right\rangle \backslash E_{j}^{\prime}$ is a union of nilmanifolds, and is a $2^{s-j}$ sheeted covering of $M^{b_{j}}$. This implies the lemma.

Let $\nu_{j}$ denote the normal bundle of $M^{b_{j}}$ in $M^{b_{j-1}}$. Here $M^{b_{0}}=M$. The following lemma describes the structure of these bundles. Let $\hat{L}$ denote the Lie algebra of $L$. Then $\hat{L}$ decomposes as an irreducible representation space of a $G$-action. Since $G$ is finite abelian, the dimension of these irreducible subspaces is at most 2 . Let $\hat{L}_{q}$ denote the direct sum of those irreducible subspaces on which $G$ acts as one of the primitive $2^{q}$-roots of unity. Note that for $k \geq 2$ we can put a $\mathbb{C}$-vector space structure on the summands of $\hat{L}_{k}$ so that the $G$ action on it is by multiplication by one of the primitive $2^{k}$-roots of unity.

Lemma 3.3. The bundle $\nu_{j}$ can be identified with $E_{j} \times_{G} \hat{L}_{s-j+1}$.

Proof. Let us first consider the case $j=1$.

Note that $\nu_{1}$, the normal bundle of $M^{b_{1}}$ in $M$, can be obtained by looking at the normal bundle of its pre-image $E_{1}$ in $\tilde{M}$, and going $\bmod G$ action. Recall that $E_{1}=\bigcup_{n \in N} p\left(E_{1}^{(n)}\right)$. It is a fact that the normal bundle of $E_{1}$ in $\tilde{M}$ is trivial (see 2], Lemma 2.2). For a component of $E_{1}$ given by $p\left(E_{1}^{(n)}\right)$, we can identify the normal bundle of $p\left(E_{1}^{(n)}\right)$ in $\tilde{M}$ with image of the normal bundle of $E_{1}^{(n)}$ in $L$. Since $E_{1}^{(e)}=L^{\alpha_{1}}$, the normal bundle of $E_{1}^{(e)}$ can be identified with $E_{1}^{(e)} \times \hat{L}_{s}$. Since $E_{1}^{(n)}$ is a translate of $E_{1}^{(e)}$, the same identification can be made for the normal bundle of $E_{1}^{(n)}$ in $L$. Hence the normal bundle of $E_{1}$ can be identified with $E_{1} \times \hat{L}_{s}$. This says that $\nu_{1}$ can be identified with $E_{i} \times{ }_{G} \hat{L}_{s}$. 
For $\nu_{j}, j>1$, just as in the $E_{1}$ case the normal bundle of $E_{j}^{\prime}$ in $N^{\prime} \backslash L$ can be identified with $E_{j}^{\prime} \times \hat{L}_{s-j+1}$. Hence the normal bundle of $E_{j}$ in $N \backslash L$ (being the pullback of the normal bundle of $E_{j}^{\prime}$ in $\left.N^{\prime} \backslash L\right)$ can be identified with $E_{j} \times \hat{L}_{s-j+1}$. This implies that $\nu_{j}$ can be identified with $E_{j} \times_{G} \hat{L}_{s-j+1}$.

Let us write $\nu_{j}=E_{j} \times_{G} \hat{L}_{s-j+1}$ as $\bigoplus_{t} E_{j} \times_{G} \mathbb{C}(t), j \leq s-1 ; \mathbb{C}(t)$ is the one dimensional $\mathbb{C}$ vector space which occurs in $\hat{L}$ and on which $G$ acts as one of the primitive $2^{s-j+1}$-roots of unity. Recall that for $j>1, E_{j}$ is a disjoint union of $E_{j}^{k}$ 's, and each $E_{j}^{k}$ is the union of components of $E_{j}$. Furthermore, since each of these $E_{j}^{k}$ are invariant under the covering action of $G$, it follows that $M^{b_{j}}$ is a disjoint union of $q\left(E_{j}^{k}\right)$, and each $q\left(E_{j}^{k}\right)$ is a union of components of $M^{b_{j}}$. There is an involutive action of $\bar{b}_{j+1}$ on $E_{j} \times_{G} \mathbb{C}(t)$, which covers the action of $\bar{b}_{j+1}$ on $M^{b_{j}}$. This is defined as follows:

$$
\bar{b}_{j+1}([x, z])=\left[\bar{b}_{j+1} x, \alpha_{j+1}^{2 k-1} z\right], \quad x \in E_{j}^{k}, z \in \mathbb{C}, \quad k=1, \ldots, 2^{j-1} .
$$

It is easy to check that this action is well defined and covers the involutive action of $\bar{b}_{j+1}$ on $M^{b_{j}}$.

Lemma 3.4. For $j \leq s-1$ eigenbundles of $\bar{b}_{j+1}$ the actions on $\nu_{j}$ are complex vector bundles.

Proof. It is sufficient to prove it for the subbundle $E_{j} \times_{G} \mathbb{C}(t)$. Note that since $\bar{b}_{j+1}$ acts as involution on the vector bundle $E_{j} \times_{G} \mathbb{C}(t)$, it decomposes as +1 and -1 eigensubbundles over the fixed point set $M^{b_{j+1}}$. Recall that $M^{b_{j}}=\bigcup_{k=1}^{2^{j-1}} q\left(E_{j}^{k}\right)$. Let $p \in q\left(E_{j}^{k}\right)$ for some $k$. Then for $[x, z]$ in the fiber over $p$ we have $\bar{b}_{j+1}([x, z])=$ $\left[\bar{b}_{j+1} x, \alpha_{j+1}^{2 k-1} z\right]$. Let $e(j, k)=k+2^{j-1}$. Since $p$ is a fixed point of $\bar{b}_{j+1}$, it follows that $p \in q\left(E_{j+1}^{k}\right)$ or $q\left(E_{j+1}^{e(j, k)}\right)$. Suppose $p \in q\left(E_{j+1}^{k}\right)$. Then we claim that there is no non-zero vector $[x, z] \in \nu_{j}(p)$ with eigenvalue -1 . We prove it by contradiction. Suppose there exists $[x, z] \in \nu_{j}(p)$ such that $\bar{b}_{j+1}([x, z])=[x,-z]$. This means $\left(\bar{b}_{j+1} x, \alpha_{j+1}^{2 k-1} z\right)=g^{l}(x,-z)$ for some $l$. Since $x \in E_{j+1}^{k}, \quad \bar{b}_{j+1} x=\alpha_{j+1}^{2 k-1} x$. This implies $g^{l}=\alpha_{j+1}^{2 k-1}$. But then $\alpha_{j+1}^{2 k-1} z=\alpha_{j+1}^{2 k-1}(-z)$. This implies $z=0$.

If $p \in q\left(E_{j+1}^{e(j, k)}\right)$, then we claim that we cannot have both \pm 1 eigenspaces. We again show this by contradiction. Let $[x, z]$ be such that $\bar{b}_{j+1}[x, z]=\left[\bar{b}_{j+1} x, \alpha_{j+1}^{2 k-1} z\right]$ $=[x, z]$. The same argument as above gives $\alpha_{j+1}^{2 e(j, k)-1} z=\alpha_{j+1}^{2 k-1} z$. This implies that $\alpha_{j+1}^{2^{j}} z=g^{2^{s-1}} z=z$. On the other hand if $[x, w]$ were a -1 eigenvector, then we would get $g^{2^{s-1}} w=-w$, but this is not possible since in this case the $G$ action on the $\mathbb{C}$ part is by primitive $2^{s-j+1}$-roots of unity.

Now we address our main question regarding bounding of $M$. By Theorem 2.1 of Stong mentioned in the Preliminaries, $M$ will bound if $M^{b_{1}}$ together with its normal bundle $\nu_{1}$ bounds. Recall that $M^{b_{2}}=q\left(E_{2}^{1}\right) \cup q\left(E_{2}^{2}\right)$. Let $\nu_{1}{ }^{+}$and $\nu_{1}^{-}$be the eigenbundles of $\nu_{1}$ with respect to the $b_{2}$ action. Applying Theorem 2.2 to the bundle space $\left(M^{b_{1}}, \nu_{1}\right)$ with $\bar{b}_{2}$-action, we see that the pair $\left(M^{b_{1}}, \nu_{1}\right)$ will bound if $\left(q\left(E_{2}^{1}\right), \nu_{21}, \nu_{11}{ }^{+}, \nu_{11}{ }^{-}\right)$and $\left(q\left(E_{2}^{2}\right), \nu_{22}, \nu_{12}{ }^{+}, \nu_{12}{ }^{-}\right)$bound separately as bundle spaces. Here $\nu_{1 j}{ }^{+}, \nu_{1 j}{ }^{-}$are restrictions of $\nu_{1}$ on $q\left(E_{2}^{j}\right), j=1,2$, respectively. Note that by Lemma $3.4 \nu_{11}{ }^{+}$and $\nu_{12}{ }^{-}$are $\mathbb{C}$ vector bundles whereas $\nu_{11}{ }^{-}$and $\nu_{12}{ }^{+}$are rank- 0 vector bundles. $\bar{b}_{3}$ has an obvious action of each of the bundle 
spaces using $(*)$; and note that fixed point set of the $\bar{b}_{3}$ action on $q\left(E_{2}^{1}\right)$ equals $q\left(E_{3}^{1}\right) \cup q\left(E_{3}^{3}\right)$ (disjoint union). We can apply Theorem 2.2 again in this case. An important point to note is that because of Lemma 3.4 all the eigenbundles obtained will be $\mathbb{C}$ vector bundles. Applying Theorem 3.3 successively (the fixed point set of $\bar{b}_{j+1}$ on $q\left(E_{j}^{k}\right)$ equals $\left.q\left(E_{j+1}^{k}\right) \cup q\left(E_{j+1}^{e(j, k)}\right)\right)$, we get that $M$ will bound if the bundle space $\left(q\left(E_{s}^{k}\right), \nu_{s k}\right)$ together with all the eigenbundles $L_{t}(s, k)$ bounds for each $k=1, \ldots, 2^{s-1}$. Here $\nu_{s k}$ denotes the restriction of $\nu_{s}$ to $q\left(E_{s}^{k}\right)$.

Note that from Lemma $3.3 \nu_{s}=\bigoplus E_{s} \times_{G} \mathbb{R}$, where $G$ acts on $\mathbb{R}$ as -1 , where the number of summands is equal to the number of 1-dimensional representation spaces of $\hat{L}$ on which $G$ acts as -1 . Let $\eta=E_{s} \times_{G} \mathbb{R}$. Let $w_{1}(\eta)$ denote the first Stiefel-Whitney class of $\eta$. The following lemma is important.

Lemma 3.5. $w_{1}(\eta)^{2}=0$.

Proof. Note that $\eta=E_{s} \times_{G} \mathbb{R}$, where $G$ acts on the first factor as covering action and on the second factor by $\mathbb{Z}_{2^{s}} \longrightarrow \mathbb{Z}_{2}$, and then $\mathbb{Z}_{2}$ acts on $\mathbb{R}$ as $\{+1,-1\}$. We can also write $\eta=E_{s}^{\prime} \times_{\mathbb{Z}_{4}} \mathbb{R}$, where $E_{s}^{\prime}=\left(g^{2^{s-2}}\right) \backslash E_{s}$ and $g$ is the generator of $G$. $\mathbb{Z}_{4}$ acts by $g$ on $E_{s}{ }^{\prime}$ and on $\mathbb{R}$ as $\{+1,-1\}$. If $s=2$, then $E_{s}=E_{s}{ }^{\prime}$. This tells us that $\eta$ is a flat $\mathbb{Z}_{4}$ bundle and hence corresponds to a map $\eta: M^{b_{s}} \longrightarrow B \mathbb{Z}_{4}$, which corresponds to some element of $H^{1}\left(M^{b_{s}}, \mathbb{Z}_{4}\right)$. This element maps to $w_{1}(\eta)$ under the map induced by $\mathbb{Z}_{4} \longrightarrow \mathbb{Z}_{2}$. Using this fact and the long exact sequence

$$
\ldots \longrightarrow H^{1}\left(M^{b_{s}}, \mathbb{Z}_{2}\right) \longrightarrow H^{1}\left(M^{b_{s}}, \mathbb{Z}_{4}\right) \longrightarrow H^{1}\left(M^{b_{s}}, \mathbb{Z}_{2}\right) \stackrel{S q^{1}}{\longrightarrow} H^{2}\left(M^{b_{s}}, \mathbb{Z}_{2}\right) \longrightarrow \ldots
$$

we conclude that $S q^{1}\left(w_{1}(\eta)\right)=w_{1}(\eta)^{2}=0$. Here $S q^{1}$ is the Steenrod square map.

Let $\nu_{s}=\eta^{l}$. We need the following lemma to finish our proof.

Lemma 3.6. The rank of $\nu_{s}(=l)$ is even.

Proof. Note that $l$ is the number of 1 dimensional vector subspaces of $\hat{L}$ on which $G$ acts as -1 . Let $\hat{L}^{-}$be the direct sum of these 1 -dimensional spaces. Since $L$ is a 2-step nilpotent Lie group and $Z(L)$ is one dimensional, we have the following bilinear map given by the Lie bracket [,]:

$$
[,]: \hat{L}^{-} \times \hat{L}^{-} \longrightarrow \mathbb{R} \text {. }
$$

Since this form is skew-symmetric, we just need to show that [,] is non-degenerate restricted to $\hat{L}^{-}$. We prove it by contradiction. Let $x(\neq 0) \in \hat{L}^{-}$be such that $[x, y]=0$ for every $y \in \hat{L}^{-}$; then we claim that $x \in \hat{Z}(L)$. This will imply that $x=0$, since $G$, acts trivially on $\hat{Z}(L)$. Let $\hat{L}^{ \pm i} \subset \hat{L}$ denote the sum of two dimensional irreducible representation spaces. Let $z \in \hat{L}^{ \pm i}$. Then some even power of the generator $g$, say $g^{2 k}$, acts as -1 on $z$. Since $L$ is 2 -step, $[x, z] \in \hat{Z}(L)$. And since $G$ acts trivially on the center, $g^{2 k}[x, z]=[x, z]$. But $g^{2 k}[x, z]=\left[g^{2 k} x, g^{2 k} z\right]=$ $[x,-z]=-[x, z]$. This implies $[x, z]=0$ for every $z \in \hat{L}^{ \pm i}$. This implies that $x \in \hat{Z}(L)$.

Now we show that the bundle space $\left(q\left(E_{s}^{k}\right), \nu_{s k}, L_{t}(s, k)\right)$ bounds. Let us write $W_{j}$ 's for the complex line bundles which occur as part of the eigenbundles $L_{t}(s, k)$. It is sufficient to show that $\nu_{s k}$ and the $W_{j}$ bound, since $q\left(E_{s}^{k}\right)$ is a nilmanifold (Lemma 3.2) and hence parallelizable, and therefore its Stiefel-Whitney classes vanish. 
Since $l$ is even and $w_{1}(\eta)^{2}=0, w\left(\nu_{s k}\right)=1$. Hence we just need to show that the $W_{j}$ 's bound. Since the $W_{j}$ 's are complex line bundles, they are orientable, and hence $w_{1}\left(W_{j}\right)=0$ for all $j$. Now any monomial in the $w_{2}\left(W_{j}\right)$ 's which is of total degree equal to the dimension of $q\left(E_{s}^{k}\right)$ is in the image of a monomial in the first Chern classes $c_{1}\left(W_{j}\right)$ under the map induced by $\mathbb{Z} \longrightarrow \mathbb{Z}_{2}$. Since the $W_{j}$ 's are flat bundles, the $c_{1}\left(W_{j}\right)$ 's are torsion elements in $H^{2}\left(q\left(E_{s}^{k}\right), \mathbb{Z}\right)$, and hence the corresponding monomial is a torsion element in $H^{m}\left(q\left(E_{s}^{k}\right), \mathbb{Z}\right), m$ being the dimension of $M^{b_{s}}\left(=\operatorname{dim} q\left(E_{s}^{k}\right)\right)$. But $q\left(E_{s}^{k}\right)$ is orientable; hence $H^{m}\left(q\left(E_{s}^{k}\right), \mathbb{Z}\right)$

has no torsion. This says that any monomial in the Chern classes of the $W_{j}$ 's of degree $m$ must vanish. Hence the image of the monomial also vanishes.

This completes our proof of Theorem 1.6.

\section{ACKNOWLEDGMENT}

The result of this paper is one part of the author's Ph.D. thesis. The author would like to thank his advisor, Professor Tom Farrell, for helping him.

\section{REFERENCES}

1. P. E. Conner and E. E. Floyd, Differential periodic maps, Springer, Berlin, 1964. MR 31:750

2. F. T. Farrell and S. Zdravkovska, Do almost flat manifolds bound?, Michigan Math. J. 30 (1983), 199-208. MR 85j:57059

3. M. W. Gordon, The unoriented cobordism classes of compact flat Riemannian manifolds, J. Diff. Geometry, 15 (1980), 81-90. MR 82a:57029

4. M. L. Gromov, Almost flat manifolds, J. Differential Geom. 13 (1978), 230-241. MR 80h:53041

5. G. Hamrick and D. C. Royster, Flat Riemannian manifolds are boundaries, Invent. Math. 66 (1982), 405-413. MR 83h:53051

6. C. Kosniowski and R. E. Stong, $\mathbb{Z}_{2}^{k}$-actions and characteristic numbers, Indiana Univ. Math. Journal, 28, (1979), 725 - 743. MR 81d:57027

7. A. I. Mal'cev, On a class of homogeneous spaces, Izv. Akad. Nauk SSSR Ser. Mat. 13 (1949), 9-32; English transl., Amer. Math. Soc. Transl. (1) 9 (1962), 276-307. MR 10:507d MR 12:589e

8. M. S. Raghunathan, Discrete Subgroups of Lie Groups, Springer-Verlag, Berlin-HeidelbergNew York, 1972. MR 58:22394a

9. R. E. Stong, Equivalent bordism and $\mathbb{Z}_{2}^{k}$ actions, Duke Math. J. 37 (1970), 779-785. MR 42:6847

Department of Mathematical Sciences, SUny at Binghamton, Binghamton, New York 13902-6000

Current address: Bloomberg L. P., 499 Park Avenue, New York, New York 10022

E-mail address: sdhar@math.binghamton.edu 